\title{
A Study on Moving Mesh Finite Element Solution of Phase-Field Models for Hydraulic Fracturing
}

\author{
Fei Zhang, Weizhang Huang, Xianping Li, and Shicheng Zhang
}

\begin{abstract}
A moving mesh finite element method is studied for the numerical solution of a phase-field model for brittle fracture and hydraulic fracture. In the phase-field modeling, a continuous phase field variable is introduced to describe the unbroken or broken status of the material, which can model fractures without explicitly tracking discontinuous displacement fields. It has the advantages of being able to handle complex cracks, crack propagation, and creation of new cracks more easily. It is noted that the parameter $l$, which describes the width of smeared cracks, should be chosen small for the model to be sufficiently accurate. On the other hand, the mesh size $(h)$ should be chosen small typically satisfying $h \ll l$ or at least $h<l$. This deems it necessary to use mesh adaptation for an efficient numerical simulation. Moreover, cracks propagate under continuous load, which means the mesh must adapt to the evolving cracks dynamically. In this talk we will employ the moving mesh partial differential equation approach for dynamic mesh adaption. Numerical examples will be presented to show that the moving mesh finite element method is able to adaptively capture the crack propagation and handle multiple crack systems.
\end{abstract}

Index Terms-Brittle fracture, hydraulic fracture, phase-field model, moving mesh, mesh adaptation.

\section{INTRODUCTION}

In the recent years, tight oil and shale gas play an important role in energy security. Shale reservoirs typically have ultra-low permeability, low porosity, and large diversity of gas occurrence. These features make shale gas or oil extraction extremely difficult and often require large-scale hydraulic fracturing technologies to achieve economic development. One critical process to increase the reservoir stimulated volume and thus improve ultimate recovery is to dilate and aggressively shear natural fractures and then connect them with the main hydraulic fracture to form a fracture network. One powerful tool to understand the physical principles behind those technologies is numerical simulation.

The existing models can be roughly categorized into two groups, discrete crack models and continuum crack models. In the former group, cracks are described as moving boundaries and represented along edges of mesh elements. One major challenge for those models is to track the moving discontinuity of the displacement field caused by the

Manuscript received February 17, 2018; revised March 30, 2018.

Fei Zhang and Shicheng Zhang are with College of Petroleum Engineering, China University of Petroleum - Beijing, 18 Fuxue Road, Changping, Beijing 102249, China (email: fzhang_cup@outlook.com, zhangsc@cup.edu.cn).

Weizhang Huang is with Department of Mathematics, the University of Kansas, Lawrence, Kansas 66049, U.S.A. (whuang@ku.edu).

Xianping Li is with Department of Mathematics and Statistics, University of Missouri - Kansas City, 5120 Rockhill Road, Kansas City, Missouri 64110, U.S.A. (lixianp@umkc.edu). evolution of cracks. On the other hand, continuum crack models treat cracks as smeared regions and do not rely on explicit description of creaks.

The phase-field modeling is a commonly used type of continuum crack model. In the phase-field modeling, a phase-field variable $d$, which depends also on a parameter $l$ describing the actual width of the smeared cracks, is used to indicate where the material is cracked and where the material is undamaged. Distinctive features of the modeling include the following. First, sharp but smooth interfaces (instead of discontinuities) are now introduced into the displacement field. Secondly, fracture initiation and propagation are completely determined by a coupled system of partial differential equations based on the energy functional. Lastly, generation and propagation of complex fracture networks do not require explicitly keeping track of fracture interfaces.

Since it was first proposed by Bourdin et al. [1], [2], the phase-field modeling for brittle and hydraulic fracture has extracted considerable attention and significant progress has been made; e.g., see [3]-[10]. Nevertheless, solving those models efficiently and robustly remains a challenging task.

The parameter $l$ that describes the width of smeared cracks, is required to be very small in order to obtain reasonably accurate solutions. Moreover, the $\Gamma$-convergence of the energy functional of the phase-field model requires the mesh size $h$ to satisfy $h \ll l$ [7], which is difficult to fulfill when uniform meshes are used. The best strategy is to use mesh adaptation which places more mesh points around localized features while using less points in other regions. Moreover, cracks can propagate, and new cracks can be created. Thus, dynamic mesh adaptation strategies should be used to concentrate mesh points around those evolving features.

We plan to employ the MMPDE moving mesh method for dynamic mesh adaptation. The MMPDE method, developed by Huang and his co-workers (e.g., see [11], [12]), is specially designed for time dependent problems and amenable to the numerical solution of fracture problems. Compared to other moving mesh methods, it has several advantages including (a) it works for any convex or concave domain; (b) the mesh is guaranteed to stay non-singular (and thus no crossing or tangling); (c) it is relatively simple to implement; and (d) it works with a large class of objective functions for mesh adaptation and quality control.

\section{PHASE-FIELD APPROACH}

\section{A. Brittle Fracture}

We first describe the phase-field modeling for brittle fracture. Denote the displacement vector by $u$. Then the strain tensor is given by

$$
\epsilon=\frac{1}{2}\left(\nabla u+\nabla u^{T}\right)
$$


For isotropic material without damage, the elastic energy density due to strain is given by Hooke's law, i.e.,

$$
W_{e}(\epsilon)=\frac{\lambda}{2}(\operatorname{tr}(\epsilon))^{2}+\mu \operatorname{tr}\left(\epsilon^{2}\right)
$$

where $\lambda$ and $\mu$ are the Lame constants. The stress tensor is related to the strain tensor by

$$
\sigma \equiv \frac{\partial W_{e}}{\partial \epsilon}=\lambda \operatorname{tr}(\epsilon) I+2 \mu \epsilon
$$

The variation of the total energy $\mathcal{W}=\int_{\Omega} W_{e} d x$ is

$\delta \mathcal{W}=\int_{\Omega} \frac{\partial W_{e}}{\partial \epsilon}: \delta \epsilon d x=\int_{\Omega} \sigma: \delta \epsilon d x=\int_{\Omega} \sigma: \epsilon(\delta u) d x$

The weak formulation becomes

$$
\int_{\Omega} \sigma: \epsilon(\delta u) d x=0 .
$$

If we add the body force vector $\mathrm{f}$, we have

$$
\int_{\Omega} \sigma: \epsilon(\delta u) d x=\int_{\Omega} f \cdot \delta u d x
$$

The boundary conditions can be defined by prescribing the surface traction $\bar{t}$ or displacement $\bar{u}$ :

$$
\left.\sigma \cdot n\right|_{\partial \Omega_{t}}=\bar{t},\left.\quad u\right|_{\partial \Omega_{u}}=\bar{u},
$$

where $\partial \Omega_{t} \cap \partial \Omega_{u}=\emptyset$ and $\partial \Omega_{t} \cup \partial \Omega_{u}=\partial \Omega$.

We now consider the situation with the presence of cracks. Denote the set of cracks by $\Gamma$. Then, the total fracture energy can be expressed as

$$
\mathcal{W}_{c}=\int_{\Gamma} g_{c} d S
$$

where $g_{c}$ is the fracture toughness which is the amount of energy needed to create a unit area of fracture surface.

The phase-field modeling uses a phase-field variable $d(x, t)$, which depends also on a parameter $l$ describing the actual width of the smeared cracks. (The dependence on $l$ is omitted for notational simplicity.) This function is smooth, has the range in $[0,1]$, and is considered as a smeared characteristic function of $\Gamma$ which is 0 or close to 0 near the cracks and 1 away from the cracks (see Fig.1(a)). The total fracture energy is approximated by the smeared total fracture energy

$$
\mathcal{W}_{c}^{l}=\int_{\Omega} \frac{g_{c}}{4 l}\left((d-1)^{2}+4 l^{2}|\nabla d|^{2}\right) d x .
$$

The elastic energy also needs to be modified in presence of cracks. To describe this, we define the decomposition of a scalar function $\mathrm{f}$ as

$f=f^{+}+f^{-}, \quad f^{+}=\max (f, 0)=\frac{|f|+f}{2}, \quad f^{-}=\min (f, 0)=\frac{f-|f|}{2}$.

For a symmetric tensor $\varepsilon$ with the eigen-decomposition $\epsilon=Q \operatorname{diag}\left(\lambda_{1}, \ldots, \lambda_{n}\right) Q^{T}$, we define

$$
\epsilon=\epsilon^{+}+\epsilon^{-}
$$

where

$\epsilon^{+}=Q \operatorname{diag}\left(\lambda_{1}^{+}, \ldots, \lambda_{n}^{+}\right) Q^{T}, \quad \epsilon^{-}=Q \operatorname{diag}\left(\lambda_{1}^{-}, \ldots, \lambda_{n}^{-}\right) Q^{T}$. $\varepsilon^{+}$is the tensile strain components which contribute to the damage process resulting in fracture while $\varepsilon^{-}$is the compression strain components which do not contribute to the damage process resulting in fracture. A commonly used model, which is inspired by damage models where a degradation function $g=g(d)$ is used to describe the reduction of the stiffness of the bulk of the solid, is given by

$$
W_{e}=g(d) \Psi^{+}(\epsilon)+\Psi^{-}(\epsilon)
$$

where

$$
\Psi^{+}(\epsilon)=\left[\frac{\lambda}{2}\left(\operatorname{tr}(\epsilon)^{+}\right)^{2}+\mu \operatorname{tr}\left(\left(\epsilon^{+}\right)^{2}\right)\right], \quad \Psi^{-}(\epsilon)=\left[\frac{\lambda}{2}\left(\operatorname{tr}(\epsilon)^{-}\right)^{2}+\mu \operatorname{tr}\left(\left(\epsilon^{-}\right)^{2}\right)\right] .
$$

The degradation function is required to satisfy the following properties:

$\begin{cases}g(0)=0: & \text { Damage occurred for } d=0 \text { and this part should vanish; } \\ g(1)=1: & \text { No damage occurs for } d=1 ; \\ g^{\prime}(0)=0: & \text { No more changes after the fully broken state; } \\ g^{\prime}(1) \neq 0: & \text { The damage has to be initiated at the onset. }\end{cases}$

A commonly used degradation function is $g(d)=d^{2}$. Thus, the total energy is

$\mathcal{W}^{l}=\int_{\Omega}\left(\left(d^{2}+k_{l}\right) \Psi^{+}(\epsilon)+\Psi^{-}(\epsilon)+\frac{g_{c}}{4 l}\left((d-1)^{2}+4 l^{2}|\nabla d|^{2}\right)\right) d x$,

where $k_{l} \geq 0$ is a (small) regularization constant. The corresponding weak formulation for $u$ and $d$ reads as

$$
\begin{aligned}
& \int_{\Omega} \sigma: \epsilon(\delta u) d x=0 \\
& \int_{\Omega}\left[\left(\frac{\partial g}{\partial d} \mathcal{H}+\frac{g_{c}(d-1)}{2 l}\right) \delta d+2 g_{c} l \nabla d \cdot \nabla \delta d\right] d x=0 .
\end{aligned}
$$

where the stress tensor is given by

$$
\sigma=\frac{\partial W}{\partial \epsilon}=\left(g(d)+k_{l}\right)\left(\lambda \operatorname{tr}(\epsilon)^{+} I+2 \mu \epsilon^{+}\right)+\left(\lambda \operatorname{tr}(\epsilon)^{-} I+2 \mu \epsilon^{-}\right) .
$$

Note that $\Psi^{+}(\epsilon)$ has been replaced by

$$
\mathcal{H}=\max _{s \leq t} \Psi^{+}(\epsilon)(s)
$$

to ensure crack irreversibility in the sense that the cracks can only grow.

\section{B. Pressurized Hydraulic Fracturing Models}

Currently there exist two types of hydraulic fracturing models, pressurized [3] and fluid-filled [10] ones. As a first attempt, we focus on pressurized hydraulic fracturing models; see Fig. 1(b). For this type of models, the pressure distribution, $\mathrm{p}_{\mathrm{f}}$, is assumed to be prescribed on cracks. We need to consider the effects of porosity since shale reservoirs are porous media. Then, the total energy is given by

$\mathcal{W}=\int_{\Omega \backslash \Gamma}\left(W_{e}-\frac{1}{2} \alpha_{B} p_{r} \nabla \cdot u\right) d \Omega+\int_{\Gamma} g_{c} d S-\int_{\partial \Omega_{t} \cup \Gamma} \tau \cdot u d S$

where $\alpha_{\mathrm{B}} \in[0,1]$ is Biot's coefficient, $\mathrm{p}_{\mathrm{r}}$ is the reservoir pressure, $\partial \Omega_{\mathrm{t}}$ is the part of boundary associated with Neumann's boundary conditions, and $\tau=\sigma \cdot \mathrm{n}$ with $\mathrm{n}$ being the unitary outward normal to $\Gamma$. We have $\sigma \cdot \mathrm{n}=\mathrm{p}_{\mathrm{f}} \mathrm{n}$ on $\Gamma$, and

$$
-\int_{\Gamma} \tau \cdot u d S=-\int_{\Gamma}(\sigma \cdot n) \cdot u d S=\int_{\Gamma} p_{f} n \cdot u d S
$$


Then, we can rewrite the total energy as

$\mathcal{W}=\int_{\Omega \backslash \Gamma}\left(W_{e}-\frac{1}{2} \alpha_{B} p_{r} \nabla \cdot u\right) d \Omega+\int_{\Gamma}\left(g_{c}+p_{f} n \cdot u\right) d S-\int_{\partial \Omega_{t}} \tau \cdot u d S$

For the pressure terms in the phase-field approach we follow [3]:

$$
\int_{\Gamma} p_{f} n \cdot u d S \longrightarrow \int_{\Omega} p_{f} \nabla d \cdot u d \Omega
$$

The phase-field model for the above energy reads as

$$
\begin{aligned}
\mathcal{W}_{l}= & \int_{\Omega}\left[\left(g(d)+k_{l}\right)\left(\Psi^{+}(\epsilon)-\frac{1}{2} \alpha_{B} p_{r} \nabla \cdot u\right)+\Psi^{-}(\epsilon)\right] d \Omega \\
& +\int_{\Omega} \frac{g_{c}}{4 l}\left((d-1)^{2}+4 l^{2}|\nabla d|^{2}\right) d \Omega+\int_{\Omega} p_{f} \nabla d \cdot u d \Omega-\int_{\partial \Omega_{t}} \tau \cdot u d S .
\end{aligned}
$$

Taking the variation, we can obtain a weak formulation for $u$ and $d$.

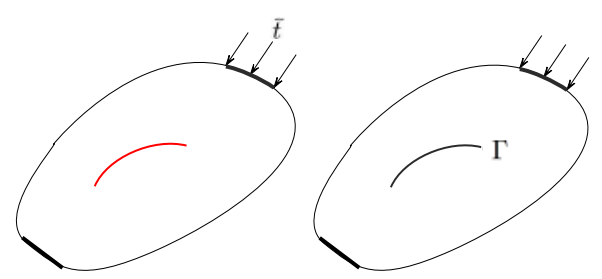

(a) brittle fracture by the phase-field approximation (b) pressurized fracture Fig. 1. A sketch of the problem setting for brittle and pressurized hydraulic fracturing models.

\section{ADAPTIVE FinITE ELEMENT SOLUTION}

In this section we describe the adaptive finite element solution of the phase-field models for brittle fracture and hydraulic fracturing using the MMPDE moving mesh method. The basic idea of variational mesh adaptation is that mesh generation and adaptation are determined by a coordinate transformation, i.e.,

$$
x=x(\xi, t): \Omega_{c} \rightarrow \Omega \Longleftrightarrow x_{j}(t)=x(\xi, t), j=1, \ldots, N_{v}
$$

This can be obtained as a minimizer of a functional:

$$
I[\xi]=\int_{\Omega} G(\mathbb{J}, \operatorname{det}(\mathbb{J}), \mathbb{M}) d x,
$$

where $\mathbb{J}=\frac{\partial \xi}{\partial x}$ : Jacobian matrix, $\mathbb{M}=\mathbb{M}(x)$ : metric tensor which provides the information needed to determine size, shape, and orientation of mesh elements. The objective of the MMPDE moving mesh method is to generate an adaptive mesh as a uniform one in the metric tensor $\mathbb{M}$. Such an $\mathbb{M}$ -uniform mesh satisfies the equidistribution and alignment conditions (e.g., see [12], [13]),

$$
\begin{array}{ll}
\sqrt{\operatorname{det}(\mathbb{M})} \operatorname{det}(\mathbb{J})^{-1}=\int_{\Omega} \sqrt{\operatorname{det}(\mathbb{M})} d x, & \forall x \in \Omega \\
\frac{1}{2} \operatorname{tr}\left(\mathbb{M}^{-1} \mathbb{J}^{T}\right)=\operatorname{det} \sqrt{\operatorname{det}\left(\mathbb{J M}^{-1} \mathbb{J}^{T}\right)}, & \forall x \in \Omega
\end{array}
$$

Then, an energy functional based on these conditions can be rewritten as (e.g., see [14])

$$
\begin{aligned}
I[\xi]= & \theta \int_{\Omega} \sqrt{\operatorname{det}(\mathbb{M})}\left(\operatorname{tr}\left(\mathbb{J} \mathbb{M}^{-1} \mathbb{J}^{T}\right)\right)^{p} d x \\
& +(1-2 \theta) 2^{p} \sqrt{\operatorname{det}(\mathbb{M})}\left(\frac{\operatorname{det}(\mathbb{J})}{\sqrt{\operatorname{det}((M))}}\right)^{p} d x
\end{aligned}
$$

where $0<\theta \leq \frac{1}{2}$ and $p \geq 1$ are two dimensionless parameters. We then follow the MMPDE approach [15], [16] and define the moving mesh equation as a gradient flow of $I[\xi]$ :

$$
\frac{\partial \xi}{\partial t}=-\frac{P}{\tau} \frac{\delta I}{\delta \xi}
$$

where $\tau$ is a positive parameter for adjusting the time scale of mesh movement and $P$ is a balancing function used to make the MMPDE to have some desired invariance properties. Since we have

$$
\mathbb{J} \frac{\partial x}{\partial t}+\frac{\partial \xi}{\partial t}=0
$$

Combine the two equations into a single one. We can get the mesh velocity $\dot{x}$

$$
\dot{x}=-\mathbb{J}^{-1} \frac{\partial \xi}{\partial t}=-\frac{P}{\tau} \mathbb{J}^{-1} \nabla \cdot\left(\frac{\partial G}{\partial \mathbb{J}}+\frac{\partial G}{\partial \operatorname{det}(\mathbb{J})} \operatorname{det}(\mathbb{J}) \mathbb{J}^{-1}\right)
$$

As mentioned above, The metric tensor $\mathbb{M}$ is the key to the success of the MMPDE method since it provides the information of the size, shape, and orientation of mesh elements needed in the adaptive mesh movement. In our computation, we choose the metric tensor based on the Hessian of the phase-field variable

$$
\mathbb{M}=\operatorname{det}(I+|H(d)|)^{-\frac{1}{6}}(I+|H(d)|),
$$

where $H(d)$ is a recovered Hessian of $d$ and $|H(d)|=Q \operatorname{diag}\left(\left|\lambda_{1}\right|, \ldots,\left|\lambda_{2}\right|\right) Q^{T}$. Since the metric tensor is based on the Hessian of the phase-field variable $d$, the mesh elements will be concentrated in the crack regions where the curvature of $d$ is large.

\section{NUMERICAL RESUlTS}

In this section we show numerical results obtained with the moving mesh finite element method for three examples. The first example is a benchmark problem used in the existing literature to examine mathematical models for brittle fracture. The second example is chosen to test the ability of our method to handle complex cracks. The last example is used to demonstrate the capabilities of our method to deal with pressurized hydraulic fracture propagation.

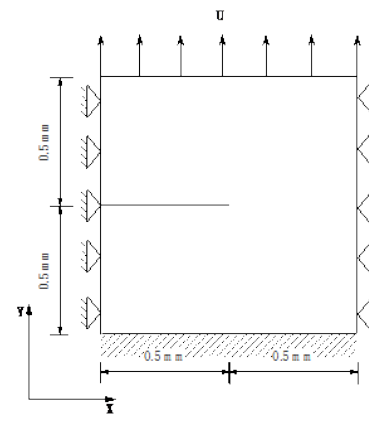

(a) tension test

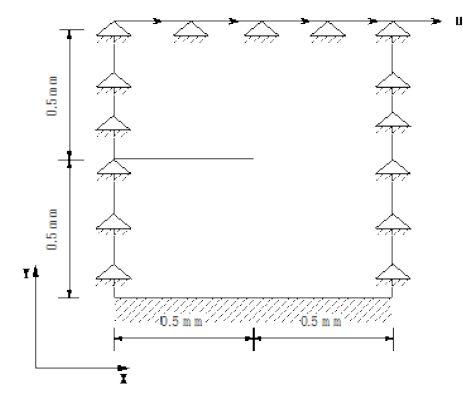

(b) shear test
Fig. 2. Geometry and boundary conditions for single edge notched specimen, (a) tension test and (b) shear test.

\section{A. Single Edge Notched Tension and Shear Test}

We first consider the single edge notched tension and shear test from Miehe et al. [8], with the geometry and boundary 
conditions being shown in Fig. 2. For the tension test, the top edge is fixed along $x$-direction while a uniform $y$ displacement $U$ is increased with time. For the shear test, the top edge is fixed along $y$-direction while a uniform $x$-displacement $U$ is increased with time. For both tests, the solid is assumed to be homogeneous isotropic with properties as elastic bulk modulus $\lambda=121.15 \mathrm{kN} / \mathrm{mm}^{2}$ and shear modulus $\mu=80.77 \mathrm{kN} / \mathrm{mm}^{2}$. The fracture toughness is chosen to $g_{c}=2.7 \times 10^{-3} \mathrm{kN} / \mathrm{mm}$.
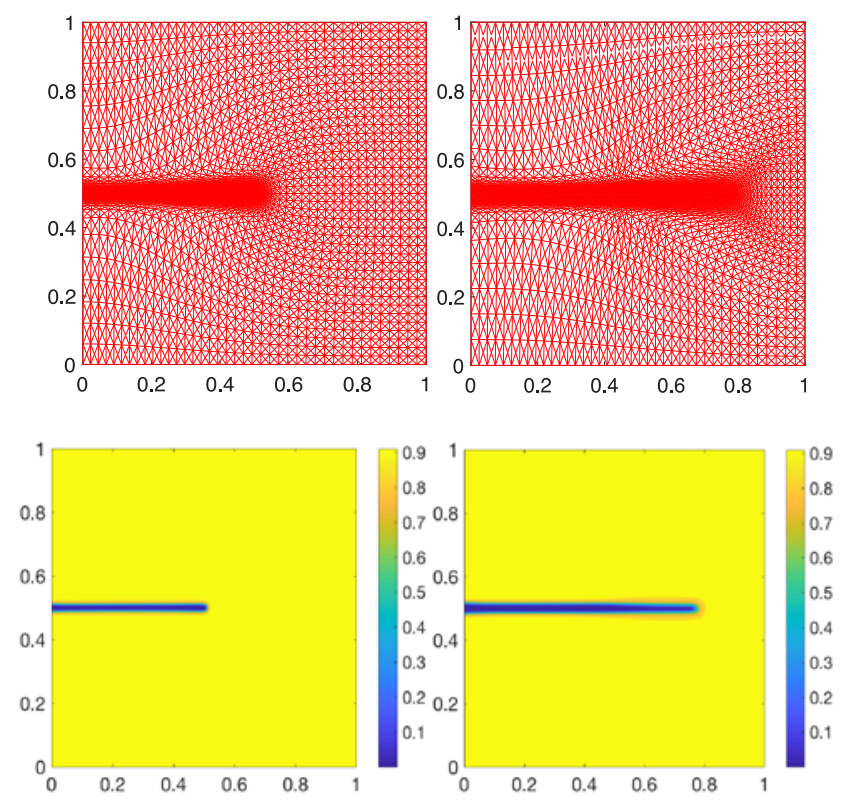

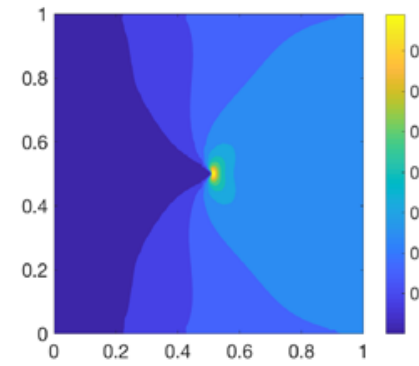

(a) $U=1 \times 10^{-3} \mathrm{~mm}$

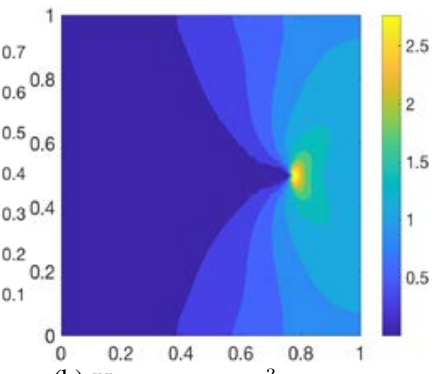

(b) $U=5.3 \times 10^{-3} \mathrm{~mm}$
Fig. 3. The mesh and contours of the phase-field and von Mises stress distribution during crack evolution for the tension test. $(\mathrm{l}=0.0075 \mathrm{~mm}, \mathrm{~N}=6,400)$.
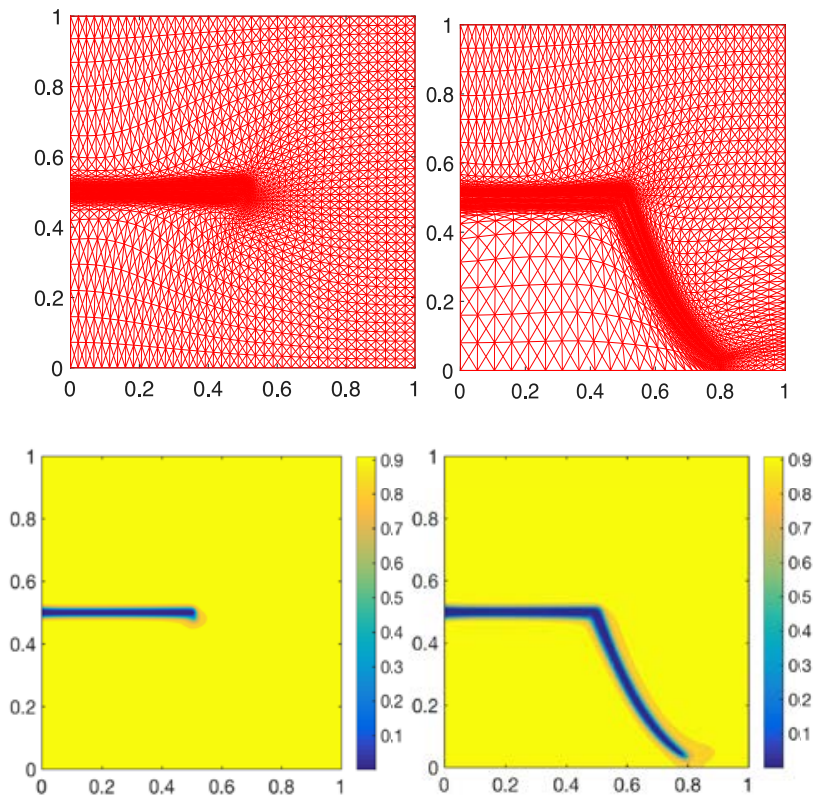

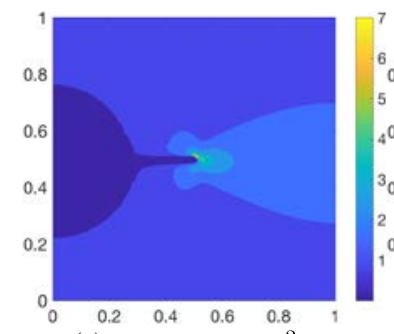

(a) $U=1.1 \times 10^{-2} \mathrm{~mm}$

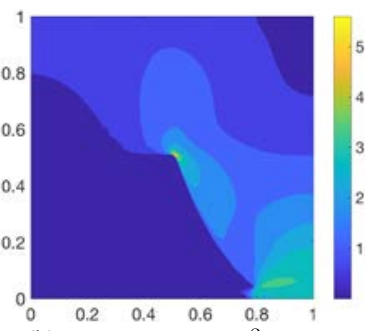

(b) $U=1.45 \times 10^{-2} \mathrm{~mm}$
Fig. 4. The mesh and contours of the phase-field and von Mises stress distribution during crack evolution for the shear test. $(\mathrm{l}=0.0075 \mathrm{~mm}, \mathrm{~N}=6,400)$.

For the tension test, two displacement increments have been chosen for the computation, $\Delta U=1 \times 10^{-5} \mathrm{~mm}$ before the fracture initiation and $\Delta U=1 \times 10^{-6} \mathrm{~mm}$ afterwards. For the shear test, we choose $\Delta U=1 \times 10^{-5} \mathrm{~mm}$. We use $l$ $=0.0075$ and a triangular mesh resulting from a rectangle mesh of size $41 \times 41$ in the computation. Typical adaptive meshes and contours of the phase-field and von Mises stress distribution during crack evolution are shown in Figs. 3 and 4 for the tension and shear tests, respectively. As we can see, for the tension test, the mesh stays symmetric near the crack for all time, while for the shear test, mesh concentration is adequate especially during the turning process of the shear crack. This property is important for handling complex crack propagation. They also demonstrate the ability of the MMPDE method to concentrate the mesh points around the cracks and follow its evolution.

For comparison purpose, the surface load vector on the top edge was introduced

$$
f=\int_{\Gamma_{\text {top edge }}} \sigma \cdot n d l
$$

where $n$ is the unit outward normal to the top edge. The load-deflection curves with a uniform mesh $(\mathrm{N}=490,000)$ and an adaptive mesh $(\mathrm{N}=6,400)$ are shown in Fig. 5 . As we can see, they are comparable with each other and agree well with the results obtained with much finer meshes in [8]. The cost between moving mesh and uniform mesh can be clearly seen in Table I.

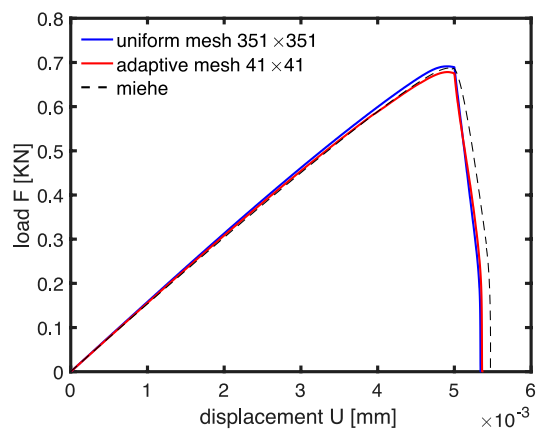

Fig. 5. The load-deflection curves for different meshes for $\mathrm{l}=0.0075 \mathrm{~mm}$ for the tension test.

TABLE I: AVERAGE CPU TIME FOR ONE TIME STEP (IN SECONDS)

\begin{tabular}{lllll}
\hline \hline Mesh size & $\begin{array}{l}\text { CPU } \\
\text { for } d\end{array}$ & $\begin{array}{l}\text { CPU } \\
\text { for } u\end{array}$ & $\begin{array}{l}\text { CPU for } \\
\text { mesh }\end{array}$ & $\begin{array}{l}\text { Total CPU } \\
\text { time }\end{array}$ \\
\hline Moving mesh 6,400 & 5.4 & 32.0 & 103.8 & 141.1 \\
Uniform mesh 490,000 & 47.9 & 1292.0 & & 1340.0 \\
\hline
\end{tabular}

Next, we consider the situation with complex cracks. The geometry and boundary conditions are shown in Fig. 6. For the three-crack and six-crack problems, the material 
parameters are the same as previous examples except $g_{c}=2.7 \times 10^{-4} \mathrm{KN} / \mathrm{mm}$ for the six-crack problem. The mesh and contours of the phase-field and von Mises stress distribution during crack evolution are shown in Figs. 7 and 8, respectively. As can been seen, the mesh points dynamically concentrate around the cracks and capture the junction process of the multiple cracks. Under continuous shear load for the three-crack problem, one of the tip for Crack 2 activates earlier due to its longer length and larger polar angle. With a closer distance, Crack 1 connects to Crack 2. Crack 3 is limited to propagation due to the interaction of other cracks. For the more complex situation under tension load with the six-crack problem (see Fig. 8), Crack 4 and Crack 3 have smaller polar angles and a closer distance, which results in the early crack merging. At later stages, Crack 3 connects to Crack 5 and the right side of Crack 5 has propagated to the edge, which leads to the whole plate to lose strength.

Finally, we employ our MMPDE moving mesh method for propagating pressurized fracture and consider two parallel fractures that interact with each other. Here, the material parameters are the same as previous examples. The fracture propagates by a given pressure that increases linearly in time, i.e., $p=t \cdot 10^{3} \mathrm{~Pa}$. We first consider a square domain with a pre-existing fracture in the middle. Fig. 9 shows the mesh and contours of the phase-field distribution during crack evolution by given increasing pressure. The fracture propagation takes place when the pressure reaches the critical propagation pressure. Next, we consider the situation with two parallel fractures. As can be seen in Fig. 10, due to the stress shadowing effect, the two parallel fractures interact with each other if the distance between them is sufficiently close. When the distance is large, influence is almost invisible; see Fig. 11.

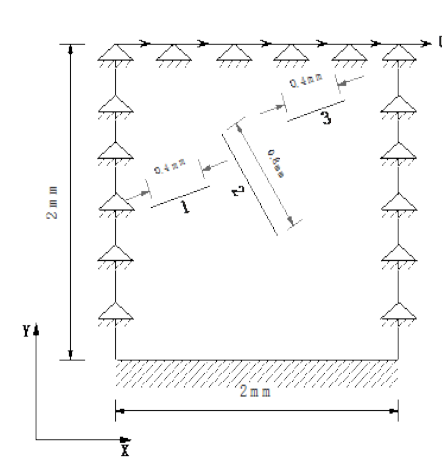

(a) three cracks

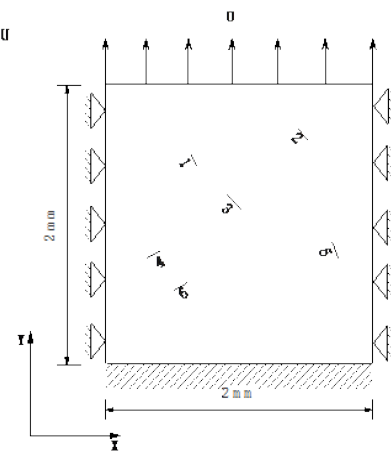

(b) six cracks
Fig. 6. Geometry and boundary conditions for the test with complex cracks, (a) three cracks and (b) six cracks.
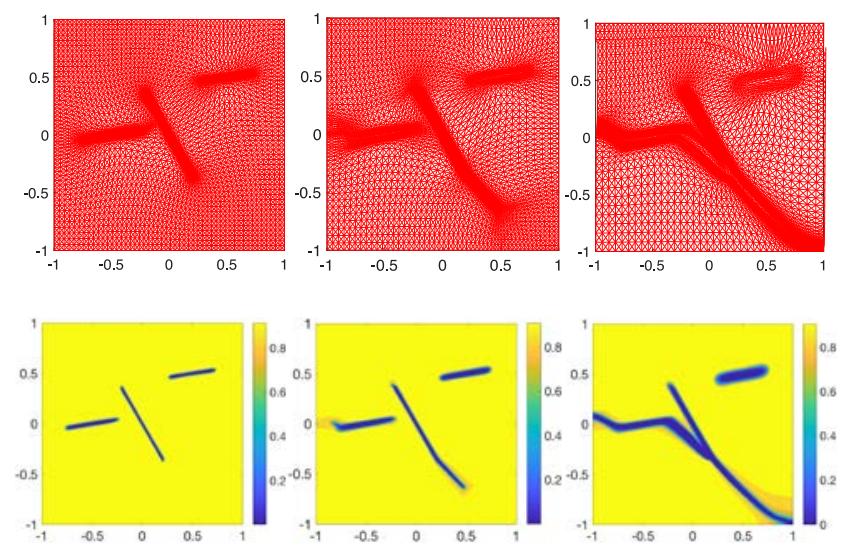

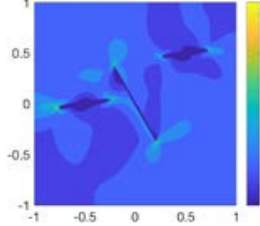

(a) $U=2 \times 10^{-3} \mathrm{~mm}$ distribution during crack evolution for the three cracks problem. $(\mathrm{l}=0.0075 \mathrm{~mm}, \mathrm{~N}=10,000)$
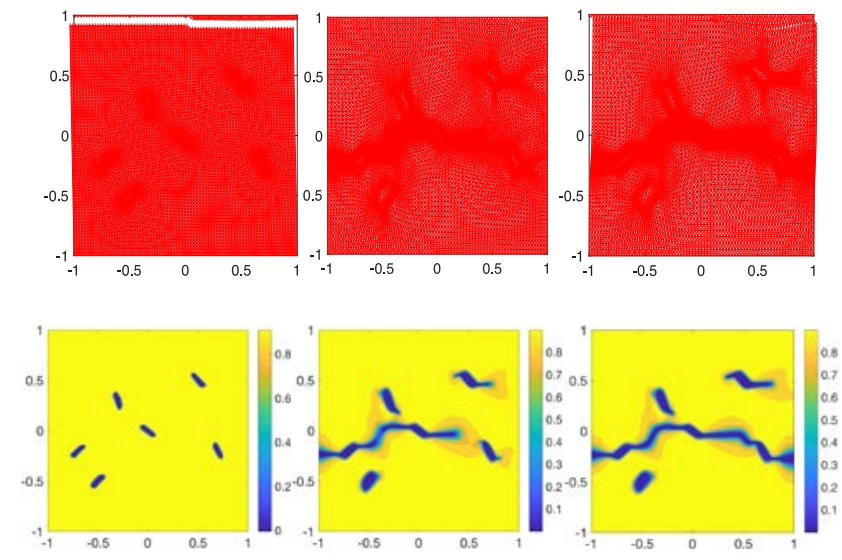

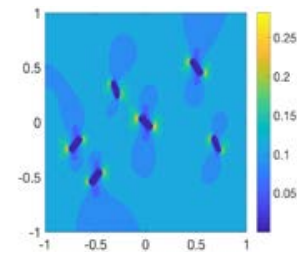

(a) $U=1 \times 10^{-3} \mathrm{~mm}$ (b) $U=7 \times 10^{-3} \mathrm{~mm}$

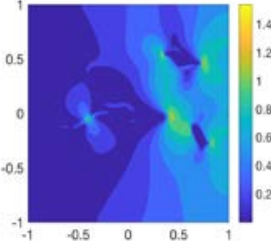

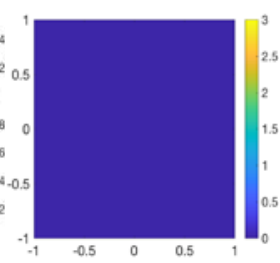

(c) $U=8 \times 10^{-3} \mathrm{~mm}$
Fig. 8. The mesh and contours of the phase-field and von Mises stress distribution during crack evolution for the six cracks problem. $(\mathrm{l}=0.0075 \mathrm{~mm}, \mathrm{~N}=40,000)$.
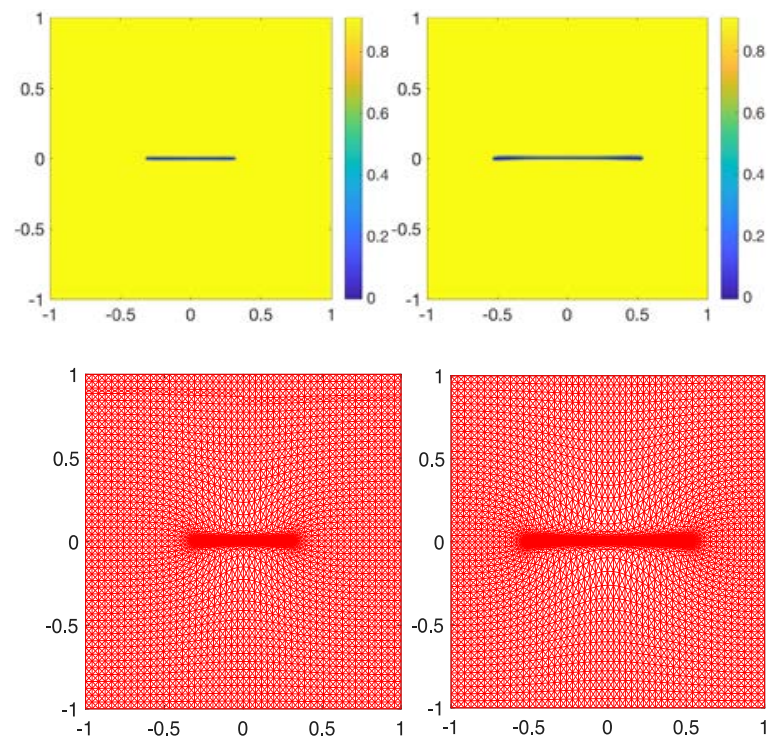

Fig. 9. The mesh and contours of the phase-field distribution during crack evolution by given increasing pressure.

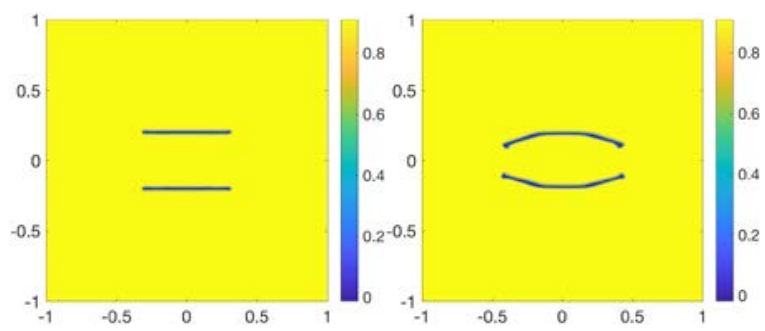




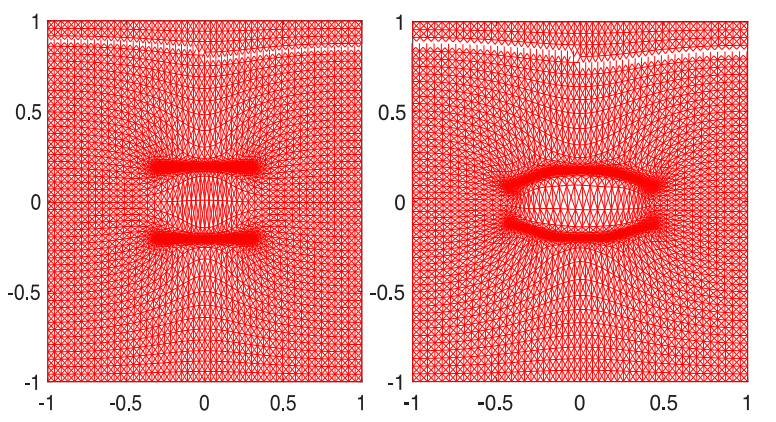

Fig. 10. The mesh and contours of the phase-field of two parallel fractures by given increasing pressure.

(distance between the fractures: $\Delta u=0.5 \mathrm{~mm}$ ).
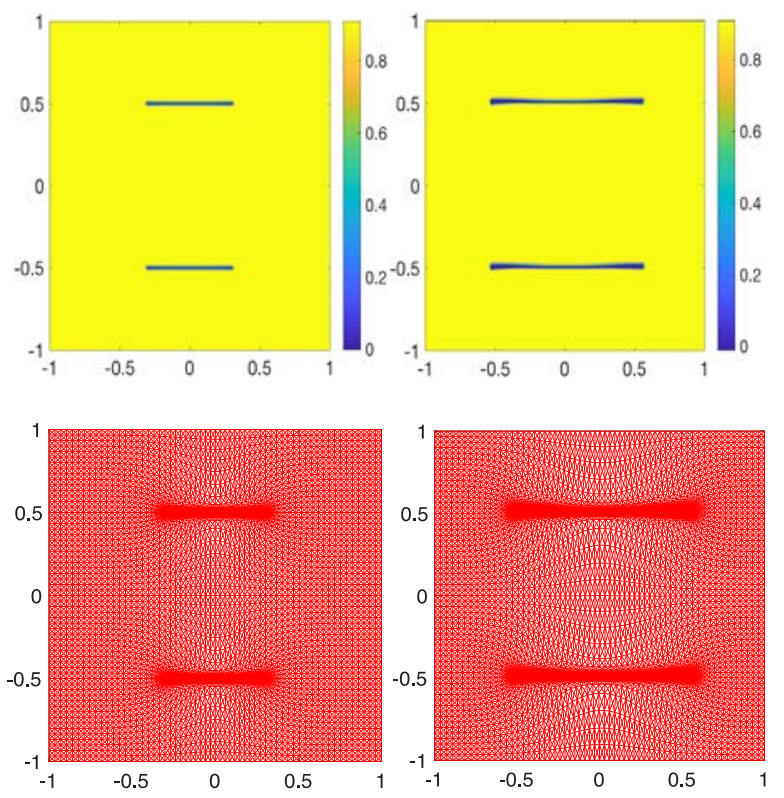

Fig. 11. The mesh and contours of the phase-field and von Mises stress distribution during crack evolution for the three cracks problem. (distance between the fractures: $\Delta u=1.0 \mathrm{~mm}$ ).

\section{CONCLUSION}

In the previous sections we have studied the moving mesh finite element solution of phase-field models for brittle fracture and hydraulic pressurized fractures. We have demonstrated that the phase-field approach has its ability to handle complex fracture including crack joining and branching phenomena. Particularly, the MMPDE moving mesh method has been used to dynamically concentrate mesh points around propagating cracks. The mesh concentration is adequate especially during the turning process of the shear cracking and stay symmetric near the crack for the tension crack. This property is important to the successful capture of crack propagation for multiple crack systems. Compared to uniform meshes, the computational efficiency is significantly improved by the moving mesh method (about 10 times faster in average in terms of CPU time for one time step).

\section{REFERENCES}

[1] B. Bourdin, G. A. Francfort, and J. J. Marigo, "Numerical experiments in revisited brittle fracture," J. Mech. Phys. Solids, vol. 48, pp. 797-826, 2000.

[2] G. A. Francfort and J. J. Marigo, "Revisiting brittle fracture as an energy minimization problem.," J. Mech. Phys. Solids, vol. 46, pp. 1319-1342, 1998.

[3] B. Bourdin, C. P. Chukwudozie, and K. Yoshioka, "A variational approach to the numerical simulation of hydraulic fracturing,"' in Proc. the 2012 SPE Annual Technical Conference and Exhibition, San Antonio, October 8-10, 2012.

[4] H. Amor, J.-J. Marigo, and C. Maurini, "Regularized formulation of the variational brittle fracture with unilateral contact: Numerical experiments.” J. Mech. Phys. Solids, vol. 57, pp. 1209-1229, 2009.

[5] M. J. Borden, T. J. R. Hughes, C. M. Landis, and C. V. Verhoosel, "A higher-order phase-field model for brittle fracture: formulation and analysis within the isogeometric analysis framework," Comput. Methods Appl. Mech. Eng., vol. 273, pp. 100-118, 2014.

[6] C. Kuhn and R. Muller, "A continuum phase field model for fracture," Eng. Frac. Mech., vol. 77, pp. 3625-3634, 2010.

[7] S. May, J. Vignollet, and R. de Borst, "A numerical assessment of phase-field models for brittle and cohesive fracture: $\Gamma$-convergence and stress oscillations," European J. Mech. A/Solids, vol. 52, pp. 72-84, 2015.

[8] C. Miehe, F. Welschinger, and M. Hofacker, "Thermodynamically consistent phase-field models of fracture: Variational principles and multi-field FE implementations," Int. J. Numer. Meth. Eng., vol. 83, pp. 1273-1311, 2010.

[9] J. Vignollet, S. May, R. de Borst, and C. V. Verhoosel, "Phase-field models for brittle and cohesive fracture," Meccanica, vol. 49, pp. 2587-2601, 2014

[10] T. Wick, G. Singh, and M. F. Wheeler, "Fluid-filled fracture propagation with a phase-field approach and coupling to a reservoir simulator," SPE J., vol. 21, pp. 981-999, 2015.

[11] C. J. Budd, W. Huang, and R. D. Russell, "Adaptivity with moving grids,” Acta Numerica, vol. 18, pp. 111-241, 2009.

[12] W. Huang and R. D. Russell, "Adaptive moving mesh methods," Applied Mathematical Sciences Series, Springer, New York, vol. 174, 2011.

[13] W. Huang, "Mathematical principles of anisotropic mesh adaptation," Comm. Comput. Phys., vol. 1, pp. 276-310, 2006.

[14] W. Huang, "Variational mesh adaptation: isotropy and equidistribution," J. Comput. Phys., vol. 174, pp. 903-924, 2001.

[15] W. Huang, Y. Ren, and R. D. Russell, "Moving mesh methods based on moving mesh partial differential equations," J. Comput. Phys., vol. 113, pp. 279-290, 1994.

[16] W. Huang, Y. Ren, and R. D. Russell, "Moving mesh partial differential equations (MMPDEs) based upon the equidistribution principle," SIAM J. Numer. Anal., vol. 31, pp. 709-730, 1994.

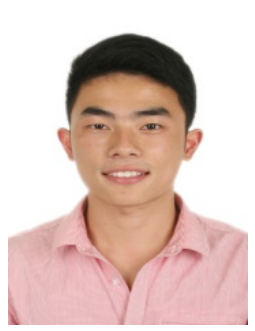

Fei Zhang was born on February 7, 1990, in HuBei, China. He had been a visiting Ph. D student in the Department of Mathematics, the University of Kansas from September 11, 2015 to September 11, 2017. Currently, he is a Ph.D student at China University of Petroleum (Beijing). His research focuses on fracture propagation mechanism and adaptive finite element solution of phase-field models for brittle fracture and hydraulic fracturing.

Weizhang Huang received his bachelor's degree from Nanjing University in

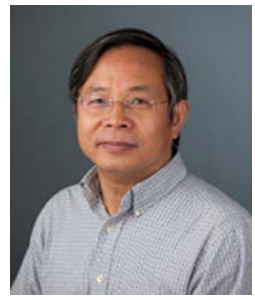
1983 and Ph.D degree from the Chinese Academy of Sciences (Institute of Applied Mathematics) in 1989. His recent research interest is in numerical analysis and scientific computing with emphasis on the numerical solution of partial differential equations. His research topics include mesh movement, mesh adaptation, anisotropic mesh generation and analysis, high order methods (collocation and spectral), geometric integration methods, and their applications. Dr. Huang is also interested in partial differential equations, dynamical systems, and fluid dynamics.

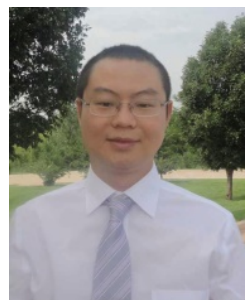

Xianping Li was born on March 16, 1979, in Hubei China. He obtained his Ph.D degree in mathematics from the University of Kansas. He is currently an assistant professor at the University of Missouri-Kansas City. His research interests include numerical solutions of partial differential equations, anisotropic mesh adaptation, and image processing. 


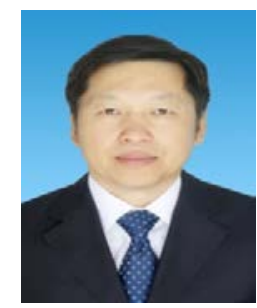

Shicheng Zhang was born in 1963, is a professor a the China University of Petroleum, Beijing. In 1986, he graduated from East-China Petroleum Institute, petroleum production engineering, and got his bachelor's degree; In 1989, he graduated from Beijing Petroleum Institute, petroleum production

engineering, and got his master's degree; In 2003, he graduated from China University of Petroleum, Beijing, oil and gas field development engineering, and earned doctor's degree. Now his research interest is theory and technology of oil and gas production engineering, hydraulic fracturing technology on unconventional oil and gas resources and reservoir numerical simulation. 